\title{
Toma de decisiones preinteractivas de profesores de educación física Planning Decisions of Physical Education Teachers
}

\author{
Maurice Piéron y Marc Cloes \\ Universidad de Liéja(Bélgica)
}

Resumen: Cuarenta y seis profesores participaron en el estudio. Entre ellos, 27 teniendo una experiencia de enseñanza de más de cinco años (12 mujeres y 15 hombres) y 19 principiantes (12 mujeres y 7 hombres). Contestaron a cinco escenarios en el marco de una entrevista requerían de decisiones a medio plazo.. Se recogieron y analizaron 434 propuestas de acción dado que una respuesta podía tener muchas propuestas diferentes. Las acciones propuestas fueron analizadas desde registros audio de las entrevistas con profesores. Fueron clasificadas de una manera inductiva. Las opiniones de los profesores con experiencia, hombres y mujeres, son más diversas que aquéllas de sus colegas principiantes, como lo atestigua el gran número de categorías diferentes. Cuatro categorías de decisiones tratan del contenido de la enseñanza: «adaptación de los ejercicios al alumno», «creación de grupos de niveles», «gradación de la dificultad», «misma actividad para todos.» Los profesores interrogados disponen inmediatamente de estrategias disponibles para tratar las diferencias individuales de los alumnos. Según sus características personales, los profesores sugieren preferentemente intervenciones específicas.

Palabras clave: Planeamiento - Decisión del profesor - Escenarios.

Abstract:: Forty-six PE teachers participated in the study. Among them, 27 had an experience of more than five years in teaching (12 women and 15 men) and 19 beginning teachers (12 women and 7 men). Teachers were presented five scenarios of instructional situations. They were asked to read each scenario and write a response detailing how they would react if the situation occurred during their teaching. Four hundred thirty-four proposals of actions were gathered and analysed since an answer could have many different proposals. The actions proposed were analysed from audiotapes of teachers' interviews. They were classified according to an inductive technique. The opinions of experienced teachers, men and women, differed than those of their beginning colleagues, as indicated by the number and content of categories. Four categories of decisions dealt with the content of education: «adaptation of the exercises to the student», «creation of levels groups», "gradation of the difficulty», and «same activity for all.» The teachers had strategies immediately available to deal with the students' individual differences. According to their personal characteristics, experienced teachers displayed a greater variety of application of sound principles of teaching..

Key words: Planning decisions - Scenario analysis.

\section{INTRODUCCIÓN}

\section{Las decisiones de planificación}

Cuando se intenta comprender los comportamientos observados en clase, abordar la reflexión, las decisiones y los valores de los participantes permite interpretar mejor lo que se observó. Este planteamiento es obviamente delicado puesto que se trata de entrar en un ámbito propenso a problemas de credibilidad.

Es crucial distinguir los valores y creencias, por una parte, y las tomas de decisiones, por otra parte. Las se refieren a dos momentos de la relación pedagógica: las decisiones preinteractivas que se toman antes de entrar en clase y las decisiones interactivas que tienen curso durante la acción donde la presentación de las tareas, el comentario o también el control del comportamiento sólo representan las principales.

En las actividades físicas y deportivas, la investigación en materia de decisiones está aún poco desarrollada. Los escasos estudios que localizamos utilizan las técnicas de recogida de los datos basados en la reflexión en voz alta o la estimulación del recuerdo. Generalmente, se desarrollan en condiciones simplificadas de enseñanza y recurren a comparaciones de distintos tipos de profesores, de tal forma que asocian las decisiones preinteractivas (programación) y las decisiones interactivas.

El estudio de Stroot y Morton (1989) se ciñe a las estrategias de planificación utilizadas por siete profesores eficaces en el nivel elemental. Su objetivo era responder a estas preguntas:

* ¿Cómo se planificaban?

* ¿Cuáles eran los factores que afectaban a dicha planificación? ¿En qué medida siguen su plan cuando se hallan ante su clase?

* ¿Existen diferencias en la planificación entre profesores principiantes, de antigüedad media o experimentados?

Fecha de recepción: 10-04-07 - Fecha de aceptación: 30-05-07

Correspondencia: Maurice Piéron

Rue des mésanges, 16

4121 Neupré - Liéja (Bélgica)

E-mail: mpieron@ulg.ac.be
* ¿Cuáles son las diferencias en la planificación de unidades consideradas flojas o experimentadas?

Todas estas observaciones nos llevan a preguntarnos si la enseñanza de la actividad física está sujeta a simple improvisación, como respuesta a acontecimientos que a menudo son imprevisibles.

Nelson (1988) desarrolló una metodología de investigación interesante para analizar las diferencias entre profesores caracterizados por niveles diferentes de pericia. Implica, en particular, el análisis de las reacciones hacia una presentación de diapositivas y de situaciones. Se presentaron varias situaciones educativas. Estas trataban del control de la clase y de las interacciones con un niño de un medio socioeconómico desfavorecido, con un muy buen deportista que tiene horror a efectuar ejercicios construidos, así como la evaluación de un niño obeso durante una unidad de enseñanza orientada hacia el desarrollo de la condición física. Los expertos respondieron con más detenimiento y con más detalles que los principiantes. Frecuentemente ha hecho referencia su lección presentada con su propia experiencia y estuvieron en condiciones mejores de encontrar un sentido a las distintas facetas del contexto. En el análisis de las situaciones, los expertos se distinguían proporcionando cerca del doble de propuestas de tratamiento del problema planteado. Sus reacciones implicaban numerosos recursos a las técnicas de refuerzo positivo.

Comparar profesores expertos y principiantes en las decisiones relativas a la planificación, utilizando las técnicas de reflexión en voz alta y la estimulación del recuerdo, contribuye a determinar los aspectos en los que se centran los profesores cuando preparan una sesión (Housner y Griffey, 1985, Griffey y Housner, 1991). Dichos autores utilizaron una situación semicontrolada, en la que los profesores de enseñanza primaria preparaban una breve sesión de iniciación a las técnicas de fútbol y de baloncesto. Durante su planificación, los profesores expertos no sólo pedían más información sobre las condiciones de enseñanza, sino que dedicaban casi el doble de tiempo para decidir qué estrategias de enseñanza utilizar. Parecían más implicados que los principiantes en la gestión de las actividades y en la información que debían proporcionar a los alumnos para facilitarles la adquisición de habilidades motrices. Sus decisiones se referían a la evaluación del nivel de los alumnos, al fee- 
dback, las demostraciones y la forma de enfocar la atención del alumno en los aspectos críticos de la realización de las tareas. Dichos resultados fueron confirmados por Howell (1987), quien apreció que durante la planificación de una unidad de enseñanza de baloncesto de cinco sesiones, los expertos analizaban las habilidades de una forma típica, que consistía en una anticipación de los errores de prestación motriz y en la búsqueda de criterios que permitieran mejorar dicha prestación. Debemos destacar que la mayoría de ellos no iniciaban su planificación por la definición de objetivos. La observación sistemática de las sesiones permitió trazar un perfil de las intervenciones y a continuación correlacionar las dos partes preinteractivas e interactivas de la sesión. El análisis de las declaraciones de los profesores muestra hasta qué punto se centran en el contenido y en la estructura de las actividades, dejando claramente en segundo plano el diagnóstico de las necesidades de los alumnos y la determinación de los objetivos de la sesión.

El estudio de Twardy y Yerg (1987) presenta numerosas analogías con el de Housner y Griffey (1985). Los autores propusieron a 30 futuros profesores de educación física un periodo de preparación (30 minutos), de una sesión de voleibol de otros 30 minutos cuyo objetivo principal era enseñar el remate. La técnica de reflexión en voz alta permitió recopilar los elementos de reflexión en los que se basaban dichos profesores. Una vez más, la estructura de la actividad constituía el punto central de la planificación. Entre 25 y 30 profesores de los 30 participantes indicaban la actividad, la analizaban, la evaluaban y consideraban su secuencia de enseñanza. Menos de la mitad de los profesores identificaban el nivel de habilidad del aprendiz o la sintonía entre las necesidades del alumno y lo que se le proponía. Un único profesor de los 30 que formaban el estudio tomaba nota de los progresos del alumno. Ninguno de ellos examinaba la concordancia entre el objetivo y la instrucción.

Los profesores dedicaron la mayor parte del tiempo de la sesión a presentar la actividad, a observar al alumno y a proporcionarle feedback. Además, esta última función era predominante entre casi dos tercios de los profesores. En el campo del comportamiento de los alumnos, la espera pasiva constituía más de un cuarto del tiempo de la sesión y, asimismo, el comportamiento predominante en dos tercios de las clases. Resulta interesante conocer que los autores hallaron relaciones significativas entre los comportamientos de los profesores en su programación y su acción en clase, en particular entre el contenido previsto, por un lado, y la demostración y presentación de las tareas, por el otro.

\section{La incidencia de la planificación en la relación pedagógica}

Graham, Hopple, Manross y Sitzman (1993) son probablemente los únicos investigadores que eligieron una situación natural para investigar las relaciones entre las decisiones de planificación y las decisiones interactivas. Dichos autores también utilizaron la comparación entre expertos y principiantes. Los datos proceden de múltiples fuentes: observación, protocolo de reflexión, notas de campo, entrevista semiestructurada, etc. Aunque el estudio sólo se basa en un número de profesores muy limitado, las conclusiones merecen una reflexión.

Los expertos:

* Se apoyan en gran medida en su experiencia para planificar las lecciones.

* Enseñan de forma diversa a partir de su conocimiento de las características de la clase y de la actitud de los alumnos en un momento dado.

* Invierten más tiempo en desarrollar las tareas a partir de la observación de las necesidades de los niños (menos tareas, mayor porcentaje de información específica y precisión).

Los principiantes:

* Se apoyan en gran medida en una documentación escrita para planificar sus sesiones.

* Utilizan la primera sesión del día para determinar si la programación del resto de las sesiones es válida o si debe ser modificada.
* Toman sus decisiones a partir de su plan escrito, más que en función de las necesidades e intereses de los alumnos.

* Cambian con frecuencia de tareas durante la actividad, en función de una necesidad aparente de hacer todo lo que estaba previsto.

A menudo, el análisis de las fases preinteractivas e interactivas de la enseñanza se hizo de forma independiente. Relacionar la preparación con la acción es de una lógica incontestable. Por el contrario, el diagnóstico relativo a los alumnos presenta poca relación con los indicadores del proceso; la presentación de las tareas y el feedback sólo presentan relaciones significativas con las decisiones preinteractivas. También se dedujo que los profesores expertos utilizaban procesos más complejos dereflexión.

El estudio de Januario (1992) sobre la relación entre las decisiones preinteractivas y los comportamientos interactivos de enseñanza es interesante. El autor utilizó la técnica de la entrevista estructurada, para analizar la reflexión del profesor y la observación sistemática de las correspondientes sesiones. La amplitud de la muestra (22 profesores y 44 sesiones) permite una generalización muy aceptable de sus conclusiones. El autor destaca el carácter interdependiente de las decisiones de los profesores. Diversas conclusiones merecen especial atención: a) el carácter intencional de la reflexión preinteractiva en el comportamiento interactivo; b) las relaciones en el interior de las dos fases preinteractivas e interactivas son más destacadas que las que existen entre las dos fases; c) las relaciones entre las variables de la fase preinteractiva son las más fuertes; d) los mejores indicadores del comportamiento en clase corresponden a la planificación más rica; e) las variables que reflejan la participación en clase son las que presentan relaciones más fuertes en ambas fases de la enseñanza; f) las preocupaciones prácticas identificadas durante la fase preinteractiva se reflejan en clase; g) el análisis de la tarea está asociada de forma significativa con la participación en las actividades de aprendizaje del alumno y h) la organización de la gestión del tiempo guarda buena relación con las variables preinteractivas.

\section{METODOLOGÍA}

Hemos decidido utilizar una técnica de análisis de escenarios. Esta metodología de investigación consiste en seleccionar varias situacionesproblemas, presentarlas a los profesores en diferentes formas tales como diapositivas, textos, videos,..., observar sus reacciones, recoger sus propuestas de acción y, finalmente, analizarlas con métodos cuantitativos y cualitativos (Nelson, 1988).

Se prepararon cinco situaciones (cuadro 1). Describen situaciones que implican la toma de decisiones a medio plazo, en las cuales una anticipación de la acción pedagógica es posible, o incluso recomendada. El primero se refiere a una situación a la cual se enfrenta a cada profesor, al compás de sus experiencias pedagógicas. Se trata de encargarse de una nueva clase al principio de año. Las otras situaciones se centran más específicamente en las decisiones que los profesores podrían tomar si se enfrentaban a alumnos que presentaban características personales particulares: nivel de elevada habilidad o baja, problemas de salud o emocionales, de morfología poco favorable a la práctica deportiva.

\section{Cuadro 1 - Escenarios propuestos}

Situaciones que implican decisiones a medio plazo (anticipación)

1. Alumno que sufre de un problema de salud, en atletismo.

2. Alumnos de niveles diferentes, en natación.

3. Alumno difícil, vive problemas familiares.

4. Alumno que sufre complejos relacionados con su fuerte corpulencia.

Cuarenta y seis profesores participaron en el estudio. Entre ellos, 27 teniendo una experiencia de enseñanza de más de cinco años (12 mujeres y 15 hombres) y 19 principiantes (12 mujeres y 7 hombres). Contestaron a ocho escenarios en el marco de una entrevista, cuatro que requerían de decisiones a medio plazo y cuatro a corto plazo. Sólo los primeros serán tratados en el artículo. Se recogieron y analizaron 434 propuestas de acción dado que una respuesta podía tener muchas propuestas diferentes. Las acciones propuestas fueron analizadas des- 
de registros audio de las entrevistas con profesores. Fueron clasificadas de una manera inductiva. (Piéron, Cloes, Klutz y col., 1997).

Sobre una muestra de 46 profesores, una media de 37 sujetos, el $80.4 \%$, indicaron haber encontrado ya las situaciones propuestas. Esto pone en evidencia la validez de los escenarios. La fiabilidad en la clasificación de las propuestas de acción de las diferentes categorías alcanza el 82.6\% de validez con el control ínter-analista y el $85.0 \%$ de validez con el control intra-analista. Se utilizó el teste de comparación de dos porcentajes propuesto por el programa de bioestadística de Glantz (1988).

\section{RESULTADOS Y DISCUSIÓN}

El porcentaje de respuestas a los escenarios utilizados alcanza el 99\%. Esto subraya que los profesores están teóricamente listos para afrontar la diversidad de sus clases. Hemos observado un número de propuestas de acción ligeramente superior en las situaciones a medio plazo (2.3 vs. 2.1). Además, son más variadas, como lo atestigua el gran número de categorías registradas para cada situación (12.5 vs. 10.5).

\section{Tomar a cargo de una nueva clase}

El primer contacto con los alumnos y las primeras lecciones que le siguen representan un momento clave en la historia y la vida de una clase. Pueden influenciar las expectativas, actitudes y comportamientos a lo largo de una unidad de enseñanza, o incluso a lo largo del año entero. Se ve también como un tipo de operación de supervivencia para los principiantes (O’Sullivan, 1990).

Durante esta etapa, el profesor debe identificar las características de sus alumnos. Esta identificación debe permitir una planificación a largo plazo de las actividades. Se fijan los objetivos a alcanzar por la clase y, en el mejor de los casos, para cada alumno o para los grupos que presentan características similares. Formulamos la hipótesis que en este momento debería comenzar la individualización o la mejor adaptación de la acción pedagógica en relación con las características de los alumnos.

Presentaremos los resultados relacionados a las decisiones tomadas por los profesores en esta situación en dos etapas. En primer lugar, intentaremos determinar qué fuentes de información explotan para este caso. En segundo lugar, nos centraremos sobre el análisis de la información recogida por los profesores.

\section{1. a. Fuentes de información explotadas por los profesores}

En conjunto, los alumnos y colegas representan la fuente de información principal de los profesores (cuadro 2). La presencia dominante de los alumnos en esta búsqueda de información marca el interés manifestado hacia ellos. Interrogar al alumnado de manera informal sobre sus características es parte de ciertas tradiciones. Esta táctica puede ser considerada como una presentación entre futuros colegas. Al inicio del año, cuando todavía no se conocen todos, las presentaciones pueden explotarse para facilitar la integración de los recién llegados.

Aparecen drásticas divergencias en la comparación entre los profesores con experiencia y los principiantes, así como entre hombres y mujeres de un mismo nivel de experiencia.

Con excepción de las profesoras principiantes, todas las otras categorías de profesores indican una búsqueda de información sobre el

\begin{tabular}{|c|c|c|c|c|}
\hline \multicolumn{5}{|c|}{$\begin{array}{l}\text { Cuadro } 2 \text { - Tomar a cargo de una nueva clase. Fuentes } \\
\text { de la información buscada }\end{array}$} \\
\hline & \multicolumn{2}{|c|}{$\begin{array}{l}\text { Profesores con } \\
\text { experiencial }\end{array}$} & \multicolumn{2}{|c|}{$\begin{array}{l}\text { Profesores eon } \\
\text { experiencia }\end{array}$} \\
\hline & $\mathrm{H}$ & $\mathrm{F}$ & $\mathrm{H}$ & $\mathrm{F}$ \\
\hline Coleats & 23.7 & 15.6 & 26,3 & 294 \\
\hline Elevados & 60.5 & 719 & 68.4 & 47.1 \\
\hline Fuente "propis" & 13.2 & 12.5 & 5.3 & 8.8 \\
\hline Administración & 2.6 & 0 & 0 & 8.8 \\
\hline Padres & 0 & 0 & 0 & 29 \\
\hline Nohav informsción & 0 & 0 & 0 & 2.9 \\
\hline
\end{tabular}

alumno en una proporción superior al 60\% de las respuestas. En los profesores con experiencia, las mujeres presentan el mayor porcentaje de respuestas, mientras que en los principiantes, los hombres resultan más representativos de manera clara.

La importancia relativa de la categoría «Colegas» acoge entreel $15 \%$ y el 30\% de las respuestas, las profesoras experimentadas representan la tasa más baja. Antes de encontrarse con sus nuevos alumnos solicitan la información con los profesores que ya tuvieron la oportunidad de trabajar con ellos. En este ámbito, los profesores de las clases de $5^{\circ}$ y $6^{\circ}$ de secundaria poseen una ventaja clara sobre los colegas que reciben a los alumnos al inicio de la escolaridad secundaria. Los adeptos a esta técnica de recogida de información parecen interesados en conocer la historia de la clase, lo que denota una voluntad en la continuidad de la acción pedagógica. Las estructuras puestas en marcha en varios centros con vista a homogenizar la enseñanza de la educación física responden a este objetivo. Este «relevo» parece constituir un indicador de eficacia. Permite un ahorro de tiempo considerable y un menor riesgo de error en la valoración de los alumnos. Ningún profesor ha señalado haber observado a los alumnos durante los años anteriores. Eso podría ser, sin embargo, un procedimiento interesante cuando el equipo pedagógico de un centro escolar es suficientemente estable.

Muy pocos profesores se dirigen a los servicios administrativos de la escuela o a los padres. En este último caso, sólo los principiantes en la profesión lo hacen. Es una pena que la información que posee la dirección y la secretaría de un centro escolar, incluso de un centro «de consejo psico-socio-medical», no sea buscada más sistemáticamente por los profesores. De hecho, esta práctica podría evitar malentendidos entre los profesores y unos alumnos. Los profesores evitan probablemente esta fuente de información por miedo a dejarse influenciar y caer en trampas como el efecto Pigmalión y el estereotipo.

Es sorprendente la ausencia de contactos entre los profesores de secundaria y los padres al hacerse cargo de una clase. Podemos plantear esta pregunta: ¿Los alumnos de mayor edad son tan independientes que la opinión de sus padres ya no es necesaria? Por otro lado, los jóvenes mismos desean quizás - la búsqueda de libertad obliga - no ver a sus padres entrometerse demasiado en su vida escolar. Es deseable que el equipo pedagógico escolar pueda recurrir más a menudo a todas las personas involucradas en la educación de los jóvenes. En este tema, el papel de los padres parece fundamental.

La comparación de las categorías con respecto al género no permite destacar tendencias preponderantes en los principiantes y los profesores con experiencia (cuadro 2). Por otro lado, los principiantes se apoyan más en sus colegas para recoger la información. Esta práctica se deriva de su estatus personal. Ellos disponen de menos experiencia y menos tiempo para reunir la información que los profesores con experiencia, generalmente ligados a una misma escuela durante varios años. En estas condiciones, los principiantes obtendrían opiniones más valiosas, ya que ellos serían los más capacitados para informarles sobre los hábitos de los alumnos. Al contrario, los profesores con experiencia, son quienes siempre disponen de alguna información referente a los jóvenes que llegan a sus clases, los principiantes carecen de referencias.

Los profesores con experiencia, hombres y mujeres, se basan más en sus propias opiniones que los principiantes. Estos están considerados más aptos para forjarse rápidamente una impresión sobre los alumnos (Clark y Peterson, 1986). Algunos parecen capaces de anticipar los problemas de disciplina que encontrarán en una clase después de su primer encuentro.

Además, hay que considerar que el contacto con los servicios administrativos de la escuela fue citado sólo por tres principiantes y un experimentado. En el caso de los primeros, podría tratarse de una búsqueda para integrarse en una estructura desconocida. Los principiantes representan el grupo de profesores que proponen la mayor variedad de fuentes de información, seis categorías. Esto podría explicarse por dos actitudes opuestas: (1) un interés muy grande hacia los alumnos y (2) un miedo bastante fuerte frente a los mismos. La literatura referente a las primeras experiencias reales de los jóvenes profesores apoyaría la segunda teoría (Schempp y Graber, 1992, Schempp, 1990, 1993). 


\section{1. b. Información buscadas por los profesores.}

La información administrativa (nombre de los alumnos, procedencia,..) y su nivel de habilidad es lo que más interesa a los profesores, del $15 \%$ al $25 \%$ en el primer caso, y del $5 \%$ al 20\% de las respuestas en el segundo. Por otro lado, diez profesores de los 46 declaraban no tomar información previa a la primera clase. Esto representa más de un quinto de la población interrogada y no ha dejado de asombrar. Varios profesores asocian esta falta de curiosidad a la idea de no dejarse influenciar por las opiniones externas. En este sentido, su decisión es comprensible. Parece muy difícil prever los objetivos y determinar las habilidades a alcanzar sin conocer el nivel inicial de los alumnos, al menos en cuanto a su motricidad. En un estudio sobre la programación hecha en condiciones normalizadas, Twardy y Yerg (1987) habían constatado que la información sobre los alumnos y sus características era menos retenida que los contenidos y los procedimientos de enseñanza.

\begin{tabular}{|c|c|c|c|c|}
\hline \multicolumn{5}{|c|}{$\begin{array}{c}\text { Cuadro } 3 \text { - Tomar a cargo de una nueva clase. Fuentes } \\
\text { de la información buscada. Tipos de información } \\
\text { buscada }\end{array}$} \\
\hline & \multicolumn{2}{|c|}{$\begin{array}{l}\text { Profesores con } \\
\text { experiencia }\end{array}$} & \multicolumn{2}{|c|}{$\begin{array}{l}\text { Profescares } \\
\text { princinian tes }\end{array}$} \\
\hline & $\mathrm{H}$ & $\mathrm{F}$ & $\mathrm{H}$ & $\mathrm{F}$ \\
\hline Información administrativa & 24,4 & 22.9 & 23.8 & 16.7 \\
\hline Nivel do habilidad & 4.9 & 20 & 4.8 & 19.4 \\
\hline Comportamientos & 12.2 & 2.9 & 4.8 & 5.6 \\
\hline Soxiceconómixo, emocional & 9.8 & 2.9 & 0 & 5.6 \\
\hline Deportes practicados & 17.1 & 11.4 & 190 & 13.9 \\
\hline Centros de interés & 7,3 & 8,6 & 19.0 & 13,9 \\
\hline Información médica & 9.8 & 5.7 & 4.8 & 11.1 \\
\hline No tay in formación & 7.3 & 5.7 & 143 & 8.3 \\
\hline Proerama de la in kerinidad & 0 & 0 & 0 & 5.6 \\
\hline Consignas a los alumnos & 0 & 17.1 & 4.8 & 0 \\
\hline Distinto & 7.3 & 2.9 & 4.8 & 0 \\
\hline
\end{tabular}

Añadiendo las respuestas propuestas como segunda o tercera elección, los profesores han totalizado 133 opciones posibles, alrededor de tres por persona. Esto significa que después de declarar no tomar ninguna información, algunos profesores cambian de opinión, pensando probablemente en la información administrativa a la cual nadie puede escapar. Esta categoría ocupa el primer rango en orden de importancia cuantitativa en todos los grupos, a la excepción de los principiantes (cuadro 2). Generalmente, la información de este tipo se refiere principalmente al nombre, apellido y domicilio. Mientras que los dos primeros puntos permiten al profesor comunicarse individualmente con cada uno de sus alumnos, el tercero le da la posibilidad de conocer su entorno social y cultural.

En la comparación según la experiencia profesional, las proporciones de tres categorías difieren en el mismo sentido en las mujeres y en los hombres. Los principiantes otorgan proporcionalmente mayor importancia a los deportes practicados y a los puntos de interés de los alumnos. Igualmente, señalan más frecuentemente no recoger información. Para las primeras dos categorías, esta constatación correspondería al interés de los principiantes de buscar medios para crear una relación afectiva positiva con los alumnos. Además, es sorprendente que los profesores que tienen menos conocimientos sobre los alumnos declaren frecuentemente no interesarse al respecto. Casi el $15 \%$ de las respuestas de los principiantes corresponden a esta opción. Es probable que la brevedad de su estancia en una escuela pueda explicar esta actitud.

La información administrativa y la información relacionada con los deportes practicados representan las mayores proporciones entre los hombres, mientras que el nivel de habilidad recibe una atención más marcada en las mujeres (cuadro 3). Como sus alumnos están más comprometidos en la práctica deportiva extraescolar (Ledent, Cloes, Telama y col.1997) se puede entender que los hombres se interesan más que sus colegas femeninos. Éstas estarían más preocupadas por determinar rápidamente si los alumnos poseen un nivel de habilidad homogéneo o no. Siendo el desarrollo físico y motriz de las chicas proporcionalmente más bajo que el de los chicos, los profesores se preocupan considerablemente para que éste intervenga enormemente en el programa de actividades. Puede considerarse que los hombres otorgan mayor interés a las informaciones susceptibles de indicarles los riesgos potenciales de problemas de disciplina («comportamiento de los alumnos» y «características socioeconómicas y afectivas» en los profesores con experiencia, «centros de interés de los alumnos» en los principiantes). Esto involucra al carácter más turbulento en los chicos, muchas veces origen de muchos incidentes de indisciplina de mayor gravedad que en las chicas (Piéron y Brito, 1990; Piéron y Emonts, 1988).

Los principiantes y las profesoras experimentadas mencionan las consignas a dar a los alumnos como parte de la información buscada. Este tipo de decisiones está ligada probablemente a la intención de poner cómodos a los alumnos en un nuevo entorno. La importancia de esta categoría en las experimentadas (el 17.1\%) corresponde al contacto afectivo que establecen más naturalmente las mujeres. Se notará, con cierto asombro, que los profesores principiantes masculinos no mencionaron nunca su condición de interinos o sustitutos (que ocupa el puesto de manera temporal) en sus respuestas.

En relación con las Propuestas de acción en respuesta a situaciones que necesitan una individualización en situaciones a medio plazo, abordaremos esta parte de los resultados en dos etapas. Primero, consideraremos cada una de las situaciones a medio plazo. Finalmente, intentaremos establecer una síntesis a partir de las diferentes situaciones.

En promedio, más de ocho de diez profesores, han declarado haber sido enfrentados a las situaciones presentadas (el 80.4\%). Esto nos autoriza a pensar que su elección fue juiciosa. De esta forma, el análisis de las propuestas de acción por los profesores entrevistados presenta un real interés práctico.

Ninguna diferencia significativa ha sido puesta en evidencia entre los profesores con experiencia y los principiantes. Es muy probable que estos últimos no sean totalmente «principiantes» en cuanto a experiencia pedagógica, pudiéndola haber ganado como educadores o en el medio deportivo, en el entrenamiento de equipos de jóvenes o incluso en el marco de prácticas de iniciación deportiva.

\section{Presencia de un alumno asmático en la clase (situación 2 -} cuadro 4)

Hemos recogido un total de 106 propuestas de acción en relación con el problema descrito en esta situación. Esto representa una media de 2,3 propuestas por profesor. Fueron repartidas en doce categorías diferentes en las que la distribución presenta algunas divergencias muy claras, según los grupos de profesores considerados.

Los principiantes proponen más propuestas de acción que los profesores con experiencia. Estos resultados van en el mismo sentido que aquellos de Cloes, Vandersmissen y Piéron (1997). Estos autores subrayaban que los profesores con experiencia tendían a identificar más

Cuadro 4 - Moclalidades de tratamiento individualizado previsto por bos profesures en el caso de la presencia de un alumno asmático

\begin{tabular}{|c|c|c|c|c|}
\hline & \multicolumn{2}{|c|}{$\begin{array}{c}\text { Pofesores oxn } \\
\text { experiencial }\end{array}$} & \multicolumn{2}{|c|}{$\begin{array}{l}\text { Profescares } \\
\text { principiantes }\end{array}$} \\
\hline & $\mathrm{H}$ & $\mathrm{F}$ & $\mathrm{H}$ & $\mathrm{F}$ \\
\hline Grupos de miveles & 9.7 & 6.9 & 20.0 & 35.5 \\
\hline Adaptación de las cieccicios & 48.4 & 27.6 & 13.3 & 12.9 \\
\hline Gradación de la dificultad & 65 & 6.9 & 26.7 & 9.7 \\
\hline Refuerzo $v$ afoctividsd & 0 & 0 & 0 & 6.5 \\
\hline Utilización de los pares & 9.7 & 3,4 & 0 & 0 \\
\hline Participación de profesor & 3,2 & 6,9 & 6.7 & 0 \\
\hline Otro bapel & 65 & 3.4 & 0 & 3.2 \\
\hline Conseios múdicos & 3.2 & 13.8 & 0 & 12.9 \\
\hline Inclu so acti vidad para kodos & 6.5 & 10.3 & 6.7 & 6.5 \\
\hline Supresión de la actividad & 3.2 & 13.8 & 6.7 & 0 \\
\hline Adsptación de la onsan żaci & 0 & 69 & 133 & 3.2 \\
\hline Varios & 3.2 & 0 & 6.7 & 9.7 \\
\hline
\end{tabular}


categóricamente que los principiantes, los problemas presentes en las secuencias de enseñanza y a seleccionar las propuestas de acción más directas.

Las opiniones de los profesores con experiencia, hombres y mujeres, son más diversas que aquéllas de sus colegas principiantes, como lo atestigua el gran número de categorías diferentes. Esta constatación parece contradecir lo que ha sido presentado anteriormente pero parece lógico, pues los profesores con experiencia han sido confrontados a una gran variedad de condiciones que les permiten enriquecer su repertorio de acciones (Calderhead, 1981).

Ocurre que la prioridad que otorgan a muchas categorías difiere bastante de aquélla de los principiantes. Así, los profesores con experiencia mencionan en proporciones más amplias la adaptación de los ejercicios al alumno y son los únicos que proponen la utilización de parejas como procedimiento de individualización en caso de problemas graves de salud. La diferencia es significativa en los hombres $(\mathrm{z}=1.986$; $\mathrm{p}=0.047)$

Los principiantes optan más a menudo por la creación de grupos de niveles. Siendo ya la diferencia muy clara entre los hombres, también es significativa entre las mujeres $(\mathrm{z}=2.373 ; \mathrm{p}=0.018)$. Estarían más preocupados por respetar la integración del alumno «diferente» dentro del grupo-clase. Entre las propuestas de acción se encuentran: el alumno asmático se une al grupo débil, trabaja en colaboración con otro profesor permitiendo una repartición de los alumnos según sus posibilidades, participa con un colega o con el profesor,..

Las diferencias observadas en la categoría «Gradación de la dificultad» reporta una utilización más fuerte por parte de los principiantes, sobre todo de los hombres. Los principiantes, hombres y mujeres, la mencionan más a menudo, subrayando su interés por la responsabilidad delalumno.

Algunas constataciones puntuales merecen ser mencionadas: la proporción importante que representan en los profesores con experiencia las categorías «Supresión de la actividad» (el 13.8\% en las mujeres y el 3.2\% en los hombres) y la presencia exclusiva de la "Atribución de otro papel al alumno diferente" (el 3.4\% en las mujeres y el 6.5\% entre los hombres). Aunque esto corresponde respectivamente a cinco y tres respuestas de 60 , es sorprendente comprobar que son los profesores con experiencia quienes recomiendan la separación del alumno concernido. Esta aproximación no refleja la imagen que se tiene habitualmente de ellos, como normalmente capaces de encontrar las propuestas de acción apropiadas al bienestar de los alumnos.

El conjunto de las diferencias identificadas, significativas o no, da la impresión que los principiantes se comprometen más al trabajo de integración. Dos hipótesis pueden explicar esta tendencia:

(1) Los profesores con experiencia han aprendido a desconfiar de las responsabilidades eventuales en materia de accidentes escolares;

(2) los principiantes dudan menos en utilizar la educación física como medio para mejorar la condición física del asmático y se preocupan más de la función de integración social de la práctica de la educación física.

Con excepción del interés más marcado en las mujeres hacia la categoría «Consejos médicos», pocas diferencias son puestas en evidencia según el género. El instinto materno de las profesoras las incitaría a «no forzar» al alumno. No olvidemos que una proporción menos importante de las chicas practican un deporte que los chicos y que aceptan más difícilmente efectuar esfuerzos penosos. Igualmente, los profesores podrían querer evitar situaciones embarazosas como son las crisis de asma en los alumnos.

\section{Alumnos con niveles de habilidad diferentes en natación (Situación 2 - Cuadro 5)}

Esta situación es aquélla para la que hemos recogido el mayor número de propuestas de acción (n=113). Cada profesor ha citado en promedio 2,5 propuestas. Esto es lógico, pues todo profesor de educación física se confronta a grupos-clase compuestos de alumnos de niveles de habilidad muy heterogéneos, particularmente en natación, donde las diferencias en el dominio y la eficiencia del nado pueden ser muy marcadas.

Cuadro 5 - Modalidades de tratami ento individualizado previsto por el profesor en el caso de grupos heterogénecs en natación

\begin{tabular}{|c|c|c|c|c|}
\hline & \multicolumn{2}{|c|}{$\begin{array}{c}\text { Profesores con } \\
\text { experiencia }\end{array}$} & \multicolumn{2}{|c|}{$\begin{array}{l}\text { Profesores } \\
\text { prin cipian tes }\end{array}$} \\
\hline & $\mathrm{H}$ & F & $\mathrm{H}$ & $\mathrm{F}$ \\
\hline Grupos de niveles & 40 & 42,9 & 40.0 & 50 \\
\hline Adartación de los ejarcicios & 17,1 & 10,7 & 0 & 3,3 \\
\hline Gradación de la dificultad & 5.7 & 7.1 & 0 & 3.3 \\
\hline Utilización de los pares & 11.4 & 14.3 & 25.0 & 23.3 \\
\hline Particinoción de motessor & 8.6 & 7.1 & 150 & 10 \\
\hline Actividad de sus tíación & 2,9 & 3,6 & 0 & 0 \\
\hline Incluso actividad para todos & 0 & 0 & 0 & 3,3 \\
\hline Adaptación de la disciplina & 5.7 & 0 & 0 & 0 \\
\hline Adaptacicin de la evaluscićn & 0 & 10,7 & 15.0 & 0 \\
\hline Adapkación de la organ ización & 5.7 & 3.6 & 5.0 & 6.7 \\
\hline Pructica extraescolar & 2.9 & 0 & 0 & 0 \\
\hline
\end{tabular}

Frente a esta diversidad, la mayoría de profesores elige crear, en primer lugar, grupos de niveles. Esta práctica respeta las consignas didácticas enseñadas en la formación inicial de los profesores y responde también a las recomendaciones del currículo oficial. Sin embargo, si los principios son los mismos, en la aplicación concreta interviene la faceta muy personal que permite la práctica de diferentes modelos de organización: tres grupos con sus tareas respectivas, agrupación de alumnos de varias clases (colaboración con otros profesores), grupos que evolucionan con respecto a sus progresos,..

Como en el primer escenario, la categoría «Adaptación de los ejercicios al alumno» constituye una solución característica de los profesores con experiencia. Esta es tres veces más importante en las mujeres que en el grupo de las principiantes (el 10.7\% vs. el 3.3\%). Entre los hombres, esta propuesta de acción sólo aparece en el grupo de los profesores con experiencia (el 17.1\%).

De nuevo, los profesores con experiencia muestran una atención muy destacada hacia el alumno considerándolo a título individual. Esta se traduce en el trabajo específico del deportista de alto nivel quien se entrenará según sus objetivos de competición. Los principiantes parecen privilegiar las relaciones interindividuales entre los alumnos. En efecto, los principiantes solicitan más a menudo que los mejores ayuden a los más débiles (el $23.3 \%$ vs. el 14.3\% entre las mujeres; el 25\% vs... el 11.4\% entre los hombres). La participación del profesor - comportamiento considerado como una manifestación de su entusiasmo hacia los alumnos (Cloes y Piéron, 1989) - es igualmente más practicada por los principiantes, pero no alcanza el umbral de estadísticamente significativa (el $10 \%$ vs. el $7.1 \%$ entre las mujeres; el $15 \%$ vs. el $8.6 \%$ entre los hombres).

Notemos, igualmente, que dos profesores con experiencia, un hombre y una mujer, han propuesto programar una actividad de sustitución (curso de salvamento) para evitar los problemas, pero sin establecer niveles diferentes. En efecto, este paso puede resolver algunas dificultades. Se puede pensar también que constituye un medio de responder al desafío de la individualización. Es asombroso que sean los profesores con experiencia, de quienes la habilidad para salvarse de las situaciones difíciles es reconocida, quienes la hayan sugerido.

La categoría «Participación del profesor» es citada proporcionalmente por más profesores masculinos (3 sobre 35 respuestas - 15 profesores -, vs. 2 sobre 28 respuestas - 12 profesores -, en Los profesores con experiencia; 3 sobre 8 vs. 3 sobre 12 en los principiantes). Confrontados a los alumnos de un nivel deportivo superior, los hombres explotarían más fácilmente esta práctica para ayudar a los más débiles.

La distribución de las principales categorías de propuestas de acción consideradas por los profesores y profesoras no difiere de manera significativa. 

dro6)

4. Alumno que vive problemas familiares (Situación 3-Cua-

Con 107 propuestas de solución, esta situación se halla en una media de 2.3 opciones por profesor interrogado. Las intervenciones de refuerzo y afectividad vienen ampliamente a la cabeza de las decisiones (del 39\% al 47\%).

Parece lógico que los profesores opten por una técnica afectiva en la gestión del problema. En efecto, las necesidades inmediatas del alumno que posee un problema se hallan sobre el plano de las relaciones humanas y los profesores parecen particularmente cuidadosos de hacerle frente. La naturaleza misma del contacto privilegiado de un profesor en el marco de las lecciones de educación física constituye un elemento de gran valor en el trato de problemas como éste.

\begin{tabular}{|c|c|c|c|c|}
\hline \multicolumn{5}{|c|}{$\begin{array}{c}\text { Cuadro } 6 \text { - Modalidades de tratamiento individualizado } \\
\text { presisto por el profesor en el caso de b presencia de un } \\
\text { alumno viviendo problemas familiares }\end{array}$} \\
\hline & \multicolumn{2}{|c|}{$\begin{array}{c}\text { Profesores eon } \\
\text { experiencis }\end{array}$} & \multicolumn{2}{|c|}{$\begin{array}{l}\text { Profesores } \\
\text { Drincipisn te: }\end{array}$} \\
\hline & $\mathrm{H}$ & $\mathrm{F}$ & H & $\mathbf{F}$ \\
\hline Refierso y a fectividad & 47.1 & 44 & 45 & 39.3 \\
\hline Adaptación de la disciplina & 23.5 & 12 & 15 & 7.1 \\
\hline Panel de modelo & 2.9 & 12 & 0 & 17.9 \\
\hline Solucion en profundidad & 29 & 8 & 0 & 7.1 \\
\hline Normas idénticas & 8.8 & 8 & 20 & 14.3 \\
\hline Información para otros & 0 & 4 & 0 & 0 \\
\hline Explotación perocomprensi vo & 8.8 & 8 & 5 & 0 \\
\hline Varios & 59 & 4 & 15 & 14.3 \\
\hline
\end{tabular}

Entre las propuestas de acción consideradas por los profesores, hemos elegido algunos ejemplos particularmente típicos: conversaciones privadas en los vestuarios, hacer comprender que el deporte ayudará a la gestión de los problemas, discusión con el alumno sobre otros asuntos que ocurren en la clase,..

Si se observa más allá de la tendencia idéntica de los profesores con experiencia y de los principiantes a intervenir sobre un plano afectivo, los dos grupos de profesores parecen adoptar propuestas de acción inversas.

Los profesores con experiencia serían más propensos a mostrarse firmes, incluso exigentes, mientras que los principiantes seguirían jugando la carta de la relación social. En efecto, los profesores con experiencia tienden a otorgar más atención a categorías tales como «Adaptación de la disciplina» (el $12 \%$ vs. el $7.1 \%$ en las mujeres; el $23.5 \%$ vs. el $15 \%$ entre los hombres), y "Mostrarse firmes pero comprensivos» (el 8\% vs. el $0 \%$ en las mujeres; el $8.8 \%$ vs. el $5 \%$ en los hombres). Precisemos que en la primera de estas categorías, el profesor se muestra severo hacia el alumno, aplicando diversas sanciones. Por otro lado, los principiantes mencionan proporcionalmente dos veces más a menudo la categoría «Reglas iguales» (el 14.3\% vs. el $8 \%$ en las mujeres; el 20\% vs. el $8.8 \%$ en los hombres).

Las tendencias registradas en los dos grupos de profesores indican que los profesores con experiencia son partidarios de propuestas de acción radicales cuando la aproximación amistosa no basta para resolver la dificultad. Los principiantes esperan influenciar el comportamiento de los alumnos-problema sin tomar en cuenta los numerosos factores externos a la escuela que los miembros del equipo pedagógico no son capaces de controlar. Una pregunta queda al aire: ¿Debe contestarse con cierta brusquedad cuando la ley del más fuerte parece ser el único lenguaje comprendido por los jóvenes que "no adquirieron los comportamientos de la vida en grupo o en sociedad» y al margen de la sociedad? Es un mensaje que podría resultar de las respuestas. Un análisis profundo sería necesario realizar con esta temática, en un estudio dedicado a responder esta pregunta. En efecto, formulamos igualmente la hipótesis que ésta conducta de los profesores con experiencia puede proceder de un malestar asociado a un sentimiento de impotencia de los profesores frente a la evolución irremediable de ciertos jóvenes y al fracaso de la mediación o la concertación.

Las profesoras parecen más centradas que sus colegas masculinos sobre el desarrollo de relaciones de confianza. Ellas otorgan más importancia a la categoría «Por un papel de modelo» que los profesores hombres. En los profesores con experiencia, esta solución representa 3 de las 25 propuestas de las mujeres, vs. 1 de las 34 de los hombres. La diferencia es todavía más clara en los jóvenes profesores donde las mujeres la citan 5 veces sobre 28 en tanto que ninguno de los 20 principiantes hombres la mencionó.

Las mujeres están, y lo hemos subrayado, más preocupadas por la calidad de las relaciones afectivas que se tejen en la clase. Esta constatación se adapta a este tipo de preocupación. Está reforzada por las diferencias encontradas en otras categorías. Observamos especialmente que:

(1) Las mujeres se centran más que los hombres en la búsqueda de propuestas de acción profundas (enviar al alumno al consejo psicosocio-medico, mantener la cohesión de grupo,...);

(2) los hombres prefieren actuar endureciendo la disciplina impuesta al alumno-problema ( $23.5 \%$ vs. $12 \%$ en los expertos; $15 \%$ vs.

7.1\% en los principiantes), o mostrándose firmes pero comprensivos, categoría citada sólo por los hombres principiantes.

\section{Alumno que sufre complejos relacionados a su fuerte cor-} pulencia (Situación 5 - Cuadro 7)

Esta situación fue vivida por la gran parte de los profesores interrogados (91.3\%). Ahora que el número de jóvenes que sufren de exceso de peso aumenta sin cesar, es lógico que los profesores de educación física se enfrenten a ello cada vez más frecuentemente. Recordemos que afecta directamente a la motricidad de los alumnos, además de los complejos psicológicos que puede engendrar. McGeorge (1991) ha ilustrado claramente la espiral nefasta a la que se arriesgan a ser arrastrados.

Como en el caso del alumno asmático, los profesores tienden a resolver el problema causado por un alumno de fuerte corpulencia adaptando los ejercicios a sus características.

\begin{tabular}{|c|c|c|c|c|}
\hline \multicolumn{5}{|c|}{$\begin{array}{l}\text { Cuadre } 7 \text { - Modalidades de tratamiento individualixade } \\
\text { peviste por el prefeser en el caso de la presencia de un } \\
\text { alumes ace maplejade que presenta una escasa inagen si }\end{array}$} \\
\hline & \multicolumn{2}{|c|}{$\begin{array}{l}\text { Profesores con } \\
\text { experien cia }\end{array}$} & \multicolumn{2}{|c|}{$\begin{array}{l}\text { Professones } \\
\text { princiobankes }\end{array}$} \\
\hline & $\mathrm{H}$ & $\mathrm{F}$ & $\mathrm{H}$ & F \\
\hline Grupes do niveles & 2.8 & 0 & 0 & 7,1 \\
\hline Adaptación de los eiascicios & 27.8 & 25.8 & 23.1 & 39.3 \\
\hline Gradación de la dificultad & 5.6 & 3.2 & 0 & 0 \\
\hline Refuerzo $\mathrm{v}$ af fect $\mathrm{w}$ idad & 13.9 & 129 & 38.5 & 21.4 \\
\hline Utilización de tos pares & 8,3 & 0 & 0 & 3,6 \\
\hline Actividad de sustiousion & 5,6 & 16,1 & 7,7 & 0 \\
\hline Conseios médicos & 2.8 & 0 & 0 & 0 \\
\hline Incluso actividad para fo dos & 2.8 & 3.2 & 7.7 & 0 \\
\hline Adaptación de la evaluación & 0 & 6.5 & 0 & 0 \\
\hline A ntisipación & 8,3 & 0 & 0 & 7,1 \\
\hline Adaptación de la orran ización & 0 & 6.5 & 0 & 0 \\
\hline Papel de modelo & 8.3 & 9.7 & 7.7 & 3.6 \\
\hline Otro panel & 13.9 & 129 & 15.4 & 17.9 \\
\hline Diverso & 0 & 32 & 0 & 0 \\
\hline
\end{tabular}

Tres categorías representan una parte importante de las propuestas de acción propuestas por todos los grupos de profesores. Estas conciernen la «Adaptación de los ejercicios», del 23\% al 39\% de las respuestas, el «refuerzo y afectividad», del 13\% al 38\% y la atribución de «otro papel», del $13 \%$ al $18 \%$. Los profesores con experiencia proponen una cuarta categoría: “actividad de sustitución» (el 16.1\% en las mujeres y el $5.6 \%$ entre los hombres). Aunque ninguna diferencia significativa sea puesta en evidencia, retendremos que los principiantes otorgan proporcionalmente más atención al refuerzo afectivo del alumno obeso. Por otro lado, la posibilidad de darle otro papel es evocado por un gran número de ellos. 
Los profesores con experiencia, hombres y mujeres, son los únicos que proponen la categoría «gradación de la dificultad.» El alumno-problema con sobrepeso parece darle menos problemas que a los principiantes. Estos últimos juegan principalmente la carta del refuerzo afectivo o evitan todo problema apartando al alumno «diferente» de las actividades, no disponiendo de referencias suficientes para proponer otras prácticas.

Ninguna diferencia se ha encontrado, ya sea que se tratara de los profesores con experiencia o de los principiantes, en cuanto a la comparación de las decisiones según el género. No es posible pues considerar que las profesoras abordan la problemática de los alumnos obesos con una táctica diferente a la de sus colegas masculinos.

Frente a las situaciones encontradas durante la lección, a las cuales necesita contestar inmediatamente, los profesores parecen más limitados en sus elecciones que cuando pueden anticipar los problemas causados por la presencia de alumnos particulares. En una situación donde los profesores disponen sólo de un margen de maniobras reducidas, reaccionarían más presionados e intervendrían para limitar la extensión de los problemas (Fernández Balboa, 1991), durante incidentes disciplinarios. Cuando los acontecimientos son previsibles, serían capaces de proponer tácticas más complejas.

\section{SÍNTESIS}

Nos limitaremos a los resultados relativos a las categorías que conciernen directamente la adaptación de las tareas propuestas a los alumnos y a aquellas acerca del aspecto afectivo. Abordaremos luego la comparación de las categorías preferidas por los profesores en función a sus características personales.

Cuatro categorías de decisiones tratan del contenido de la enseñanza: «adaptación de los ejercicios al alumno», «creación de grupos de niveles», «gradación de la dificultad», «Misma actividad para todos.»

La primera de ellas reagrupa más del 10\% de las propuestas de acción en dos situaciones de cada tipo (cuadro 2). Estas últimas se caracterizan por las dificultades físicas que uno o dos aprendices encuentran con motivo de su diferencia («problemas de salud»: 27.4\%; «complejos atados a la corpulencia»: 37.3\%; «modificación de la actividad»: 30.4\%; «alumnos de baja estatura»: 63.3\%). Si la organización de un programa individual representa la táctica más directa y juiciosa en la adquisición de habilidades motrices, ésta puede presentar riesgos para la integración del alumno diferente pues se refuerza su aislamiento.

Cuando deben tener en cuenta las características individuales en clases más numerosas, los profesores optan a menudo por la organización de las actividades en grupos de niveles (cuadro 2): "niveles de habilidad diferentes en natación” (52.4\%); “aplicación de dos grupos de niveles diferentes” (25.4\%). En este caso, el profesor prefiere más la integración de los alumnos que en las propuestas de acción propuestas anteriormente. El poder de decisión queda sin embargo a su competencia.

Es asombroso comprobar que el trabajo por fichas individuales, clasificado en la categoría «gradación de la dificultad», no representa una proporción importante de las propuestas de acción que en el caso de alumno indispuesto debido a un problema de salud: 16.8\% (cuadro 2). Este estilo de enseñanza es considerado, sin embargo, como uno de los más evolucionados por Mosston y Ashworth (1986). Este exige un gran dominio por parte de los profesores, lo mismo que de la colaboración de los alumnos. Como éste hace falta de vez en cuando, los profesores dudarían en utilizarla por miedo a perder el control de la clase.

La categoría «misma actividad para todos» reagrupó el 13.3\% de las propuestas de acción propuestas en respuesta a la situación centrada sobre la aplicación de dos grupos de niveles diferentes (cuadro 2). Si este tipo de decisión puede ser percibido como contrario a los principios de individualización, varios ejemplos de propuestas de acción que pertenecen a esta categoría ponen en evidencia que los profesores quedan atentos a las dificultades de los más débiles: «ejercicio simple para todo el mundo», «ninguna diferencia si no el alumno se siente ofendido»,..
Proponiendo este tipo de propuestas de acción, los profesores parecen evitar la frustración del alumno «diferente», generalmente más débil. Procediendo de esta manera es posible preguntarse si no se arriesgan a disminuir la motivación en los alumnos más dotados. Éstos tienen el mismo derecho de practicar a un nivel que les permita desafiarse. En el grupo de propuestas de acción sobre la acción afectiva se presentan dos categorías: «refuerzo y afectividad» y «valorización por un papel de modelo.»

La primera se revela muy importante para el equilibrio psicológico o afectivo de los aprendices. Aparece lógicamente en las situaciones "problemas familiares» (40.0\%) y «complejos atados a la corpulencia» (20.9\%), (cuadro 2). Una acción afectiva es parte igualmente de las propuestas de acción más frecuentes cuando un alumno reacciona desfavorablemente hacia la actividad, situaciones 1 y 2 a corto plazo. Esta categoría está presente entre las propuestas de acción propuestas en todos los escenarios con excepción del referente a los alumnos de diferentes niveles en natación. Los profesores otorgan mucho valor a las intervenciones afectivas en el trato de las diferencias individuales. Reservándole un lugar tan importante a este tipo de decisiones, parecen plenamente conscientes del papel fundamental del afecto en la calidad delarelación pedagógica.

Enseñar al alumno «diferente» puede ser igualmente un ejemplo positivo que representa una solución privilegiada en dos situaciones: «complejos atados a la corpulencia» (11.4\%) y «aplicación de dos grupos de niveles diferentes» (26.6\%), (cuadro 2). Poner un alumno más débil a la cabeza de un equipo, poner en evidencia sus capacidades en otras disciplinas o sus progresos, constituye otros tantos procedimientos que le permitirán sentirse integrado al desarrollo educativo y no vivir las sesiones de educación física como una carga.

El número de propuestas de acción por respuesta y el número promedio de categorías de propuestas de acción difiere poco de un grupo a otro. Las comparaciones de la importancia cuantitativa de las categorías según el género, la antigüedad y el nivel de enseñanza desprenden sólo estadísticamente un número muy limitado de diferencias significativas.

Aunque no alcanzan el umbral de estadísticamente significativas, varias se repiten de una situación a la otra. Los profesores con más experiencia otorgan más interés a la implicación personal del alumno «diferente», esto le deja la posibilidad de integrarse a las actividades. En cinco situaciones sobre seis, la categoría «Gradación de la dificultad» presenta una proporción superior en las propuestas de acción de su grupo que en la de los profesores más jóvenes. Éstos privilegian más la organización de grupos de niveles, tres situaciones sobre cinco. Puede ser que no dominen suficientemente las formas de organización más evolucionadas. Los profesores de secundaria prefieren más el papel de modelo que sus colegas de otros niveles de enseñanza, cuatro situaciones sobre seis. Proponer una misma actividad para todos los alumnos constituiría una solución más masculina que femenina, cuatro situaciones sobre seis. La categoría «refuerzo y afectividad» aparece en proporciones semejantes en todos los grupos.

\section{CONCLUSIONES Y PERSPECTIVAS}

Los profesores interrogados disponen inmediatamente de estrategias disponibles para tratar las diferencias individuales de los alumnos. Son más numerosas y diversificadas cuando es posible anticipar las decisiones. Dos grandes grupos de propuestas de acción han sido puestos en evidencia. Uno se refiere a las modalidades de adaptación de las tareas, el otro recurre al aspecto afectivo de la relación pedagógica. Según sus características personales, los profesores sugieren preferentemente ciertas intervenciones.

La eficacia de la técnica del escenario ha sido confirmada y nos incita a recomendarla en la preparación de los profesores basada en una práctica reflexiva. Cada profesor podría interrogarse regularmente sobre las decisiones que tomaría si fuera enfrentado a las situaciones-problema susceptibles de ser encontradas en la práctica. 
La utilización de la reflexión pedagógica a partir de situaciones encontradas en la realidad diaria de la clase no se limita obviamente a las situaciones presentadas en el artículo. Otras situaciones de la fase interactiva de la enseñanza exigen decisiones inmediatas del profesor para garantizar la calidad de la vida en clase, su control y a veces la integridad física de los alumnos.

La técnica de reflexión a partir de situaciones encuentra aplicación en:

1. La formación profesional de los futuros profesores de Educación física. Permite prepararlos a encontrar situaciones que plantean problemas en el practicum o en sus clases en el momento de la entrada en la profesión. Permite elegir las reacciones más convenientes a comportamientos particulares del alumnado.

2. La formación seguida. Los profesores comunican su vivido en la clase, analizan situaciones reales y hacen una elección entre varias reacciones posibles.

3. La investigación. Es posible comparar las reacciones de profesores según las situaciones, la experiencia profesional, el nivel de enseñanza de los alumnos...

\section{Referencias}

CALDERHEAD, J (1981). A psychological approach to research on teachers' classroom decision making. British Educational Research Journal, 7, 51-57.

CLARK, C., \& PETERSON, P. (1986). Teachers' thought processes. In, M Wittrock (Ed.), Handbook of Research on Teaching (3rd edition). New-York: McMillan, 255-296.

CLOES, M, \& PIERON, M (1989). Identifi-cation des comportements enthousiastes de l'en-seignant perçus par des élèves lors de séances d'éducation physique. Revue de dEducation Physique, 29, 7-16.

CLOES, M, VANDERSMISSEN, A., \& PIE-RON, M (1997). Comparison of physical education teaching events' perception by beginning and experienced teachers. In, F. Carreiro da Costa, J. Diniz, L. Carvalho, \& M. Onofre(Eds). Proceedings of the AIESEP International Seminar «Research on Teaching. Research on Teacher Education». Lisbon: Universidade Tecnica de Lisboa, 2000, 60-66.

FERNANDEZ-BALBOA, J.M. (1991). Beliefs, Interactive Thoughts, and Actions of Physical Education Student Teachers Regarding Pupil Misbehaviors. Journal of Teaching in Physical Education, 11, 58-78.

GLANTZ, Primer of biostatistics. The program. McGraw-Hill, 1988.

GRAHAM, G., HOPPLE, C., MANROOS, M., \& SITZMAN, T. (1993). Novice and experienced children's physical education teachers: Insights into their situational decision making. Journal of Teaching in Physical Education, 12, 197-214.

GRIFFEY, D., \& HOUSNER, L. (1991). Differences between experienced and inexperienced teachers' planning decisions, in-teractions, student engagement and instructional time. Research Quarterly for Exercise and Sport, 62, 196-204.

HOUSNER, L., \& GRIFFEY,D. (1985). Teacher cognition: Differences in planning and interactive decision making between experienced and inexperienced teachers. Research Quarterly for Exercise and Sport, 56, 45-53.

HOWELL, D. (1987). Planning and interactive decision making in expert elementary physical education teachers. Ph.D., Louisiana State University.

JANUARIO, C. (1992). 0 pensamento do professor. Relação entre as decisões pré-interactivas e os comportamentos interactivos de ensino em educação física. Doct. diss., Universidade Técnica de Lisboa.

LEDENT, M., CLOES, M., TELAMA, R., ALMOND, L., DINIZ, J. \& PIERON, M. (1997). Participation des jeunes Européens aux activités physiques et sportives Sport, 159/160, 61-71.

McGEORGES, S. (1991). Activités pour élè-ves obèses. Revue de l'Education Physique, 4, 187-191.
MOSSTON, M., \& ASHWORTH, S. (1986). Teaching physical education. (3rd Ed.). Columbus: C. Merrill.

NELSON, K, Thinking processes, manage-ment routines and student perceptions of experts and novice physical education teachers, Unpublished doct. diss., Louisiana St. University, 1988.

O'SULLIVAN, M(1990). Beginning teachers'struggle for legitimacy.ln, R. Telama, L. Laakso, M Piéron, I. Ruoppila, \& V Vihko (Eds.), Physical education and life-long physical activity. Jyvds-kyla: The Foundation for Promotion of Physical Culture and Health, 349 357.

PIERON, M \& EMONTS, M (1988). Analyse des problèmes de discipline dans des classes d'éducation physique. Revue de 1'Education Physique, 28, 1, 33-40.

PIERON, M, CLOES, M, LUTS, K, LEDENT, M, PIROTTIN, V, \& DELFOSSE, C. (1997). Analyse de la prise en considération des carac-téristiques individuelles des élèves dans les déci-sions et les comportements d'enseignants experts et débutants, Rapport d'une recherche réalisée dans le cadre d'une convention passée entre la Communauté française de Belgique et l'Univer-sité de Liège(157/ 96), Liège: ISEPK.

PIERON, M. \& BRITO, M. (1990). Analyse d'incidents d'indiscipline survenant dans des classes de l'enseignement préparatoire (10-12 ans). In, J. Duran, J.L. Hernandez \& L.M. Ruiz (Eds) Humanismo y nuevas tecnologias en la educación física y el deporte. Madrid: INEF, 113-117.

SCHEMPP, P. (1990). Student teaching: Day One. In, M Lirette, C. Paré, J Dessureault \& M Piéron (Eds.), Intervention en éducation physi-que et en entraînement. Bilan et perspectives. Physical education and coaching. Present state and outlook for the future. Sillery: Presses de l'Université du Québec, 144-150.

SCHEMPP, P. (1993). The effect of planning on the instructional behaviors of preservice teachers Constructing professional knowledge: A case study. Journal of Teaching in Physical Education, $13,1,2-23$.

SCHEMPP, P., \& GRABER, K. (1992). Teacher socialization from a dialectical perspective: Pretraining through induction. Journal of Teaching in Physical Education, 11, 329-348.

STROOT, S., \& MORTON, P. (1989). Blueprints for learning. Journal of Teaching in Physical Education, 8, 213-222.

TWARDY, B., \& YERG B. (1987). The impact of planning on inclass interactive behaviors of preservice teachers. Journal of Teaching in Physical Education, 6, 136-148. 